\title{
I „E-Public Sociology“ als kritische soziologische Intervention?
}

\author{
Manuel Nicklich* und Stefan Sauer ${ }^{* *}$
}

\section{Zusammenfassung}

Die Soziologie spielt im Vergleich zur Betriebswirtschaft, Volkswirtschaft und neuerdings auch Virologie im öffentlichen Diskurs eine vergleichsweise untergeordnete Rolle. Letztlich lassen sich dabei zwei Paradoxien entfalten: Die Soziologie (a) spielt in gesellschaftlichen Diskursen häufig keine Rolle, obwohl sie hierzu prädestiniert sein müsste, und (b) wenn doch, wird ihr Blick besondert. Im Zusammenhang mit der Debatte zur öffentlichen Soziologie bildet sich mittels digitaler Möglichkeiten die „E-Public Sociology“ heraus, die eine beinahe voraussetzungslose Kommunikation mit der interessierten Öffentlichkeit annimmt. Im vorliegenden Text entwickeln wir ein Vier-Felder-Schema zur Analyse soziologischen Wirkens in der Öffentlichkeit und ein darauf fußendes Kommunikationskonzept als Teil der „E-Public Sociology“. Exemplarisch für die sich durch Digitalisierung neu ergebenden Kommunikationsmöglichkeiten fokussieren wir Podcasts als spezifische Form soziologischer Tätigkeit zwischen, öffentlicher Soziologie und, Soziologie in der Öffentlichkeit'. Dabei zeigt sich, dass zur Intervention Systematischeres als die bloße Steigerung der Präsenz der Soziologie gefordert ist.

Schlagwörter: Kritische Theorie, öffentliche Soziologie, Podcasts, Zeitdiagnose

\section{“E-Public Sociology" as a Critical Sociological Intervention}

Abstract

In comparison with business administration, economy and recently virology, sociology plays a rather subordinate role in public discourse. According to this, two paradoxes unfold: (a) in the discussion of societal problems sociology seems irrelevant although the discipline can be seen as predestined in dealing with those issues and (b) if it is involved it is assigned a special rather peripheral role. In the debate about public sociology increasingly the concept of "E-Public Sociology" emerges and emphasizes the almost unconditional communication with an interested public. In our text we develop a two-by-two scheme to analyze sociological effects in public and corresponding concept of communication with a special focus on podcasts as a part of "E-Public Sociology". With a focus on podcasts as special form of sociological activity between public sociology and sociology in public., we exemplify the new possibilities induced by digital transformations and evaluate how sociological podcasts might be seen as legitimate societal intervention. We show that it is not enough to simply increase visibility of the discipline, but you need a more systematic approach.

Keywords: public sociology, critical theory, podcasts, sociological diagnosis

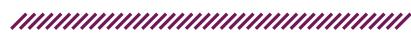

* Hauptansprechpartner; Friedrich-Alexander-Universität Erlangen-Nürnberg, Institut für Soziologie, Nuremberg Campus of Technology (NCT), Fürther Straße 246c, 90429 Nürnberg, Twitter: @soziologie_nct, E-Mail: manuel.nicklich@fau.de

** Friedrich-Alexander-Universität Erlangen-Nürnberg, Institut für Soziologie, Nuremberg Campus of Technology (NCT), FürtherStraße 246c, 90429 Nürnberg, Twitter: @soziologie_nct,_E-Mail: stefan.sauer@fau.de

${ }^{* * *}$ Dieser Text wurde teils ermöglicht durch eine Förderung der Deutschen Forschungsgemeinschaft (DFG) - Projektnummer 442171541 (DFG-Schwerpunktprogramm 2267: Digitalisierung der Arbeitswelten). Bedanken möchten wir uns zudem bei den Teilnehmer*innen der Ad-hoc-Gruppe „Podcasts in der Soziologie“ (DGS-Konferenz 2020) für das Feedback zu einer vorherigen Version des Texts sowie bei Romy Blinzler für die Unterstützung bei der Vorbereitung des Manuskripts.
} 


\section{Einleitung}

Die Soziologie ist als ,Wissenschaft von der Gesellschaft geradezu prädestiniert dazu, mit dieser in Dialog zu treten, laut eigenen Krisendiskursen (Aulenbacher et al. 2017a) spielt sie jedoch - gemessen beispielsweise an Betriebswirtschaft, Volkswirtschaft und neuerdings auch Virologie - im öffentlichen wie politischen Diskurs eine vergleichsweise untergeordnete Rolle. Nicht selten scheinen sich Soziolog*innen in einem Spannungsfeld zu befinden: eigene Motivation oder gesellschaftliche Nützlichkeitserwartung legen die Partizipation an gesellschaftlichen Diskursen nahe, soziologische Akribie - oder Pedanterie - und akademische Neutralitätsgebote erschweren die Anschlussfähigkeit an diese. Als paradigmatisch für das Spannungsfeld können die innerfachlichen Diskurse um Zeitdiagnosen (Lichtblau 2017; Neun 2016; Osrecki 2011; Volkmann 2015) und „Public Sociology“ (Burawoy 2005, 2015) gelten. Diese stellen eine „legitimationsbedürftige soziologische Gattung“ (Neun 2016: 303) dar und fristen eine „Randexistenz" (ebd.). Trotz starkem Anwachsen von zeit- und gesellschaftsdiagnostischen soziologischen Veröffentlichungen (z. B. Beck 1986; Mouffe 2018; Nassehi 2020; Reckwitz 2019; Streeck 2015) sind diese nicht als soziologisches Teilgebiet ausgewiesen, in soziologischen Verbänden wie der Deutschen Gesellschaft für Soziologie (DGS) organisational nicht vertreten und Qualität wie Rezeption höchst heterogen. Ergänzend - und mit zunehmendem Gewicht - bildet sich mittels sozialer Medien und Podcasts die „E-Public Sociology“ (Healy 2017; Schneider 2014) heraus, bei der eine - zumindest im Vergleich mit dem gedruckten Wort - beinahe voraussetzungslose Kommunikation mit der interessierten Öffentlichkeit angenommen wird.

Die Frage nach Soziologie in der Öffentlichkeit hat bereits Charles W. Mills in seinem wegweisenden Werk „The Sociological Imagination“ zum Problem der Disziplin gemacht (Mills 1959/2016), welches sich offenbar bis heute gehalten hat und anhand zweier aufeinander aufbauender Paradoxien entfaltet werden kann: Die Soziologie (a) spielt in gesellschaftlichen Diskursen häufig keine Rolle, obwohl sie hierzu prädestiniert sein müsste, und (b) wenn doch, wird ihr Blick - im Sinne Marcuses (s. u.) - besondert (s. u.), sprich: Für das Feuilleton reicht es (manchmal) - für die Partizipation an (entscheidenden) Diskursen jedoch nicht. Und so halten auch Aulenbacher et al. (2017a: 13) bezüglich der Resonanz auf soziologische Analysen fest: „Intellektuell brillant, gut formuliert und - zumindest scheinbar
- möglichst weit entfernt von dem, was im politischen Raum zur Entscheidung ansteht, kann sie mitunter selbst in Deutschland Preise gewinnen." Die Frage ist also, welche „Öffentlichkeit“ wird mit den Forschungsaktivitäten erreicht oder soll erreicht werden? Anders formuliert: In öffentlichen, zumeist bildungsbürgerlich geprägten und nicht selten moralisch aufgeladenen Diskursen findet Soziologie öffentlich statt, bei konkreten politischen Sachfragen und als kritisches Korrektiv, die breit und von Betroffenen selbst rezipiert werden, dagegen nicht - wie zur Entstehungszeit dieses Textes an der sogenannten Corona-Krise sichtbar wird. Burawoy (2015: 40) meinte wohl Ähnliches, wenn er im Anschluss an Gramsci formuliert: „Ich schlage einen anderen Ansatz vor, in dem die Soziologie nicht durch ihren bezeichnenden Gegenstand (,Gesellschaft') definiert wird, sondern durch ihren Standpunkt, der nicht der Standpunkt einer bestimmten Gruppe ist, sondern der eines bestimmten Ortes, nämlich der Zivilgesellschaft. [...] Ich beziehe mich dabei auf die Organisationen, Institutionen und Bewegungen, die einen Ort besetzen, der weder Teil der Wirtschaft noch des Staates ist." Vielmehr geht es bei den vorliegenden Fragen zur Stellung der Soziologie also um die „veränderte Wissensproduktion“ (Aulenbacher et al. 2017a: 14) und damit gerade um die intermediäre Position zwischen politischem System und den Lebenswelten der Betroffenen (Neun 2018: 17), in der die Zivilgesellschaft „ein Interesse an deren Expansion und kollektiven Selbstorganisation hat" (Burawoy 2015: 41). Bedeutsam sind demnach Aktivitäten, die die Grenzen des Wirkungsraums der bisherigen Leitmedien und damit eines „selbstreferentiellen Subsystem“ (Aulenbacher et al. 2017a: 23) gesellschaftlicher Positionseliten überschreiten. Dabei ist anzunehmen, dass gerade eine solche Öffentlichkeit zunehmend auch auf digitaler Ebene stattfindet und Formaten wie Podcasts (und Vlogs etc.) eine besondere Rolle zukommt. So sind es insbesondere Podcasts, die durch ihre Audiokommunikation und ihre performative Qualität - nicht selten in (sokratischer) Dialogform - eine besondere Vertrauenswürdigkeit (Böhle et al. 2014: 51) insbesondere auch für ,Fachfremde a aussenden können. Sie verzeichnen darüber hinaus steigende Nutzungszahlen, ${ }^{1}$ wobei die Kategorien „Nachrichten, Wirtschaft und Politik“

1 https://de.statista.com/statistik/daten/ studie/876487/umfrage/nutzung-von-podcasts-in-deutschland/ [letzter Zugriff: o8.o6.2021]. 
sowie „Wissenschaft“ die bedeutendsten darstellen. ${ }^{2}$ Es sind also Voraussetzungen geschaffen, eine so verstandene Öffentlichkeit zu erreichen, welche von der Soziologie zunehmend auch in Form von Podcasts auf unterschiedliche Art und Weise adressiert wird.

Im Folgenden stellen wir die Diskussionen im Rahmen der „Public“ (Kapitel 2.1) wie „E-Public Sociology“ (Kapitel 2.2) als unseren Ausgangspunkt dar. Hieran anknüpfend stellen wir unseren konzeptionellen Fokus vor und entwickeln, aufbauend auf einer Auseinandersetzung mit Form - d. h. über welche Kanäle die Öffentlichkeit angesprochen wird - und Inhalt $-\mathrm{d}$. h. welche Themen aufgegriffen und welche Positionen in Diskussionen tatsächlich eingenommen werden - der soziologischen Kommunikation (Kapitel 3.1) und einigen pragmatischen Gedanken zur gesellschaftspolitischen Fundierung (Kapitel 3.2) ein VierFelder-Schema zur Analyse soziologischen Wirkens in der Öffentlichkeit (Kapitel 3.3) und ein darauf fußendes Kommunikationskonzept mit besonderem Fokus auf „E-Public Sociology“ (Kapitel 3.4). Anhand einer derzeit häufig diskutierten Form des Podcastens stellen wir exemplarisch für die sich durch die Digitalisierung neu ergebenden Möglichkeiten auch die Frage nach Kriterien, mittels derer soziologische Podcasts als ,legitime gesellschaftliche Intervention gelten können, und analysieren ,Podcasten' als spezifische Form soziologischer Tätigkeit zwischen ,öffentlicher Soziologie‘ und ,Soziologie in der Öffentlichkeit'. Diese Differenzierung legen wir - mit Blick auf die (gesamte) „Public Sociology“ - anhand des Wissenstransfers in die Öffentlichkeit (Haas 2019) und des gesellschaftspolitischen Problembewusstseins dar (Neun 2018). Das heißt, es wird im Grunde mehr und Systematischeres gefordert als die bloße Steigerung der „Präsenz der Soziologie“ (Treibel/ Selke 2012: 417). Es geht vielmehr auch darum, wie Soziologie ihr (kritisches) Potenzial mit einer gewissen Konkretion und ohne zu viel außer-soziologischen Ballast nutzen kann. Podcasten mit einem Geltungsanspruch, im Sinne öffentlicher Soziologie, impliziert daher, diese Kriterien in die Kommunikation soziologischer Forschung integrieren und im Sinne einer Intervention umsetzen zu können.

2 https://de.statista.com/statistik/daten/ studie/1076171/umfrage/beliebteste-themen-bei-dernutzung-von-podcasts-in-deutschland/ [letzter Zugriff: o8.06.2021].

\section{Das Hoffen der „Public Sociology“}

2.1 „Public Sociology“ und Zeitdiagnosen als, Stiefkinder' der Soziologie?

Historisch gesehen gibt es die Diskussion zum Verhältnis von Gesellschaft und Soziologie bereits seit der Herausbildung der Disziplin (für Deutschland: Reif 2016). Einen Grund für die kontinuierliche Beschäftigung mit dem Thema sieht Neun in der Tatsache, dass Soziologie „aufgrund ihrer starken historischen Wirkung, der besonderen Relevanz ihrer Themen für ein breiteres Publikum und der hier erfolgten theoretischen Reflexionen zum Verhältnis von Wissenschaft und Gesellschaft/Öffentlichkeit, eine zentrale Position in dieser allgemeinen Debatte zur Wissenschaftskommunikation [einnimmt]" (Neun 2018: 19). Es geht somit nicht zuletzt um die Frage der öffentlichen Wahrnehmung sowie der gesellschaftlichen Relevanz der Disziplin. ,Relevanz' bezieht sich hierbei insbesondere auf die Bedeutung der Disziplin für die Gestaltung und Bearbeitung von sowie die Reaktion auf gesellschaftliche Problemlagen. Dass diese Reaktion nicht - wie oft angedacht - von Markt und Staat gelöst wird, sondern häufig jenseits dieser Sphären verhandelt und bearbeitet wird, zeigt die „Public-Sociology“-Debatte, die seit einigen Jahren in mehr oder minder starker Intensität geführt wird (Burawoy 2015).

Die Fähigkeit der Soziologie, die Stellung der Problemlöserin auszufüllen beziehungsweise die Legitimität, diese Rolle einzunehmen, wird jedoch nicht erst heute bezweifelt (Burawoy 2005; Neun 2018). Auch wenn die Debatte somit keine neue ist, wurde sie jüngst von Beiträgen zur „Public Sociology“ neu entfacht (Burawoy 2005; Jeffries 2009). Angestoßen durch die von Burawoy gehaltene Rede „For Public Sociology“ im Jahr 2004 beschäftigten sich die initialen Texte insbesondere mit dem Verhältnis der Soziologie als wissenschaftlicher Disziplin und der Zivilgesellschaft (Burawoy 2005; Jeffries 2009). Eine zentrale Idee von Burawoy ist dabei die Arbeitsteilung unterschiedlicher Formen der Soziologie, bei der er die öffentliche Soziologie und deren Notwendigkeit in den Mittelpunkt stellt, um damit auf das „Einflussdefizit der Disziplin zu antworten“ (Lamla 2014: 493). Im Anschluss an Brady (2004) hält Neun fest, dass die öffentliche Soziologie „durch zwei Merkmale [definiert werden kann]: 1. durch den Versuch, ein breites Publikum zu erreichen, und 2. dadurch, das öffentliche Wohl zu fördern“ (Neun 2018: 14). Dabei zeigt der zweite Punkt 
den grundsätzlich normativen Anspruch dieser Form der Soziologie. Unterstrichen wird dies nochmals von Burawoy (2005: 324), der für eine kritische öffentliche Soziologie "the necessity and possibility of moving from interpretation to engagement" betont und einen „critical turn“ herausstellt, „namely a turn to critique, that is a public sociology that is ,critical of ' as well as ,critical to the world it engages, a public sociology that seeks to transcend rather than uphold what exists“. Eine nicht so einfach zu bestimmende Größe ist jedoch die Frage nach jener des „öffentlichen Wohls“. Dabei kann an Vertreter*innen der Kritischen Theorie angeschlossen und sich auf grundsätzliche Werturteile bezogen werden. So weist Marcuse auf zwei Urteile hin, die er für zentral hält: einerseits, dass „das menschliche Leben lebenswert ist oder lebenswert gemacht werden kann oder sollte“, und andererseits, dass „in einer gegebenen Gesellschaft spezifische Möglichkeiten zur Verbesserung des menschlichen Lebens bestehen sowie spezifische Mittel und Wege, diese Möglichkeiten zu verwirklichen“" (1967/2014: 12). Die objektive Gültigkeit dieser Urteile zu thematisieren sei das Gebot einer kritischen Analyse, die auf empirischem Boden geführt werden solle. Ausdruck dieses als „öffentliches Wohl“ verstandenen Werts sei die „Herabminderung“ oder „Erleichterung des menschlichen Kampfes um Dasein“ (Marcuse 1967/2014: 12) (vgl. auch Kapitel 3.2).

Insbesondere vor dem Hintergrund expliziter (!) normativer Ansprüche einer wissenschaftlichen Disziplin nicht überraschend, fand die Debatte nur zögerlich Einzug auch in die deutsche Soziologie (Aulenbacher et al. 2017b; Dörre et al. 2019; Neun 2018; Selke 2020; eine Ausnahme bildet Bude 2005). Dabei fielen die Reaktionen grundsätzlich gespalten aus. Neben wohlwollenden Stimmen wurden auch Befürchtungen und Kritik artikuliert. Diese reichen über die verwendete Terminologie bis hin zu Befürchtungen von Legitimationsverlust aufgrund der moralischen Aufladung von Texten (Neun 2018: 23f.).

Von denen, die die angestoßene Debatte positiv aufgenommen haben, wird bei der Umsetzung einer öffentlichen Soziologie immer wieder die Rolle der Medien betont (Damitz 2013; Osrecki 2011). Um diese $\mathrm{zu}$ erreichen, müsse soziologisches Wissen jedoch eine spezifische Kommunikationsform annehmen, die nicht zuletzt in soziologischen Zeitdiagnosen erkannt werde (Damitz 2013; Neun 2018). Analog zu der Bewertung der öffentlichen Soziologie insgesamt wird auch gegenüber soziologischen Zeitdiagnosen eine eher gespaltene Haltung zwischen Vertreter*innen der Disziplin deutlich.
Wissenschaftler*innen „kritisieren gegenwartsdiagnostische Positionen für ihre Überpointiertheit, ihren Alarmismus und ihre oft schemenhafte Darstellung historischer Prozesse“ (Osrecki 2018: 454). Demgegenüber hält Osrecki fest, dass Verfechter*innen „gerade darin ein probates Mittel [sehen], um soziologische Argumente im öffentlichen Diskurs zu verankern und um die breite Sichtbarkeit des Faches außerhalb der akademischen Welt sicherzustellen“ (ebd.: 454). Attraktiv sind Zeitdiagnosen demnach durch ihren Zugang zu einem breiten Publikum und die Möglichkeit für Soziolog*innen, „jenseits etablierter Positionen forschen zu können“ (ebd.: 469). So müsse man den Vorwurf vielmehr umkehren und gegen die Position richten, die „diese vitalen Funktionen für das Fach übersieht und Gegenwartsdiagnosen entweder als fachliche Beiträge zu ernst nimmt oder sie vor dem Hintergrund einer idealisierten Wissenschaftsauffassung für gegenstandslos hält" (ebd.: 469). Gerade von Massenmedien sei diese Form gut aufzunehmen, da die Neuigkeit der Erkenntnis - und damit die massenmediale Substanz - im Zentrum dieser Kommunikationsform stehe (Osrecki 2011). Doch schafft allein das Zugehen der Soziolog*innen auf ein breites Publikum mit soziologischen Zeitdiagnosen kein zwangsläufiges Interesse, sondern es müssen Thesen formuliert werden, die auch in ihrem globalen Ansinnen bei einzelnen Personen verfangen (dazu auch Osrecki 2011, 2018). Es muss demnach sowohl die gesamtgesellschaftliche als auch die individuelle Bedeutung der These für möglichst viele Personengruppen herausgearbeitet werden.

\subsection{Von der „Public Sociology“ zur „E-Public Socio- $\log y^{\prime \prime}$}

Mit Blick auf das breite Publikum, das adressiert werden sollte, nimmt Osrecki die veränderten Konsumgewohnheiten in den Blick und hebt dabei insbesondere die Bedeutung der Digitalisierung hervor: Es hat „sich ein überaus punktuell lesendes Publikum entwickelt, das sich relevante Debatten weder vom Feuilleton, noch von Bücherklubs, eminenten Verlagen oder gar arrivierten Intellektuellen vorselektieren lässt. Die Digitalisierung der Medienlandschaft erlaubt stattdessen eine individuelle und punktgenaue Auswahl genau derjenigen Blogs, Foren und ,sozialen` Medien, in denen die eigenen Befindlichkeiten am besten zum Ausdruck kommen" (Osrecki 2018: 470). So wird das „Internet [als] Synonym für die weitere Ausdifferenzierung von Öffentlichkeit“" (Schwarz/Wetzel 2019: 80) 
beschrieben und auch von Aulenbacher et al. (2017a: 27) ein „neuerlicher Strukturwandel des Öffentlichen“ konstatiert und als bedeutsam für die Frage der öffentlichen Soziologie hervorgehoben.

Gemäß dieser Feststellung wurden auch im Zuge der Debatte zur öffentlichen Soziologie zunehmend Beiträge verfasst, die sich mit der Wirksamkeit von Wissenschaftskommunikation auseinandersetzen und dabei die audiovisuelle gegenüber der textbasierten Kommunikation als wirksamer hervorheben (Shrum/ Castle 2014). Mit der Thematisierung digitaler Möglichkeiten in dieser Diskussion etablierte sich mit der „E-Public Sociology“ (Healy 2017; Schneider 2014) ein Sammelbegriff für digital gestützte Formen soziologischer Wissenschaft. Insbesondere die eigentlich voraussetzungslose Kommunikation mit der Öffentlichkeit, die durch das Internet ermöglicht wird, wird in den Mittelpunkt der Erläuterungen gestellt. Verbunden mit der Digitalisierung und dem Aufkommen sozialer Medien werden insbesondere drei Aspekte hervorgehoben: eine unmittelbarere Interaktion zwischen Wissenschaft und der Öffentlichkeit, die Erhöhung der Sichtbarkeit und Rezeptionsmöglichkeiten für ein breites Publikum sowie die erhöhte Messbarkeit von Aufmerksamkeit, z. B. durch die Zählung von Klicks, Downloads usw. (Healy 2017). Dementsprechend werden die Eintrittsbarrieren als signifikant niedriger angesehen und die digital unterstützten Formen der Kommunikation als Brücke zwischen Wissenschaft und Öffentlichkeit bezeichnet (Than 2019). So ist es - nicht zuletzt aufgrund fehlender Gatekeeper - einfacher, Forschungsergebnisse sichtbar zu machen, sich an Diskussionen $\mathrm{zu}$ beteiligen und Standpunkte $\mathrm{zu}$ bestimmten Sachverhalten zu beziehen (Healy 2017; Than 2019). Healy weist jedoch auch auf die mit digitalen Kommunikationsformen verbundenen Probleme hin und unterstreicht neben missbräuchlicher Nutzung ein lediglich auf quantifizierbare Aspekte ausgerichtetes, instrumentelles Vorgehen mit dem Ziel, Rankings zu erstellen (Healy 2017: 779; kritisch dazu auch Mau 2017).

Neben den konventionellen Möglichkeiten mit der Öffentlichkeit in Austausch zu treten, hat die Digitalisierung neue Formate hervorgebracht (Than 2019). Dementsprechend wurden in der Diskussion zur „E-Public Sociology “ im Laufe der Zeit das Blogging (Wade/Sharp 2013), Podcasts (Schwarz/Wetzel 2019; Than 2019) aber auch Social Media im allgemeineren Sinne (Healy 2017) als Formate diskutiert. Diesem Trend hat sich auch die DGS nicht verschlossen und sich mit ihrem
„SozBlog“ eine digitale Form der Wissenschaftskommunikation nutzbar gemacht. Dabei wird der Vorteil des Bloggens nicht zuletzt in der Interaktion zwischen Wissenschaftler*innen mit dem Publikum, aber auch innerhalb des Publikums selbst über KommentarSpalten gesehen (Wade/Sharp 2013). Oftmals als Vorteil begriffen, wird das Unmittelbare des Bloggens auch als problematisch betrachtet, da etwaige Fehler auch direkt für ein breites Publikum sichtbar werden (Wade/Sharp 2013). Doch gerade auch mit Blick auf Podcasts wird die Hoffnung eines „Feuilleton[s] von unten“ gehegt (Schwarz/Wetzel 2019: 78). Nicht zuletzt verweisen die Autoren Schwarz und Wetzel (2019) auf die ,neuen Gesetzmäßigkeiten im Vergleich mit traditionellen Medien, da bei Podcasts Format, Publikum und - aufgrund der oftmals dezentralen Organisation - Eigentumsverhältnisse eine geringere Rolle spielen. Auch sei das Format insgesamt offen und es gehe oftmals vielmehr „um den Prozess selbst und um die Erfahrungen, die man beim Senden macht" (2019: 82). Allerdings werden den Wissenschaftler*innen in diesen Formaten auch andere Fähigkeiten abverlangt und Than hält in Bezug auf Podcasts fest: „[...] podcasts require a completely different skills set: story telling, conversational reaction with the host, some humor" (Than 2019). Das ist ein Grund, warum sich Wissenschaftler*innen gemäß Than (2019) nach wie vor eher für konventionelle oder dann eben Blogs oder soziale Medien als Formate öffentlicher Soziologie entscheiden. Podcasts wird darüber hinaus jedoch nicht nur die Rolle der effizienten Vermittlung von soziologischem Wissen zugeschrieben, sondern auch ein Potenzial hinsichtlich der Produktion von Erkenntnis. Es bestehe durch das Podcasten eine grundsätzliche Chance für die Disziplin: „Die Soziologie kann sowohl für sich selbst als auch für die demokratische Öffentlichkeit glaubwürdig sein, wenn sie ein offenes Verhältnis zur eigenen Performativität entwickelt" (Schwarz/Wetzel 2019: 84).

\section{Reflexionen (un)berechtigter Hoffnung}

\subsection{Zurück zu Inhalt und Kommunikation}

Angesichts dieser Diskussionen stellt sich jedoch die Frage, worüber gesprochen wird, wenn im Zusammenhang von Podcasten der Begriff der öffentlichen Soziologie bemüht wird. Entsprechend der anfangs formulierten Paradoxien bewegt sich die Soziologie als Disziplin zwischen öffentlicher Selbstvergewisserung und (intendierten) gesellschaftlichen Wandlungspro- 
zessen. Wobei es scheint, als ob in der Debatte, wie das eine oder auch das andere zu bewerkstelligen ist, eine Verschiebung von Fragen des Inhalts zu Fragen der Form stattgefunden hat. Das heißt, dass bei der Intention, das Einflussdefizit der Disziplin zu reduzieren, vor allem Aspekte der Wissenschaftskommunikation und Aufbereitung der Ergebnisse in den Mittelpunkt rückten, während Fragen der inhaltlichen Bedeutung für die Gesellschaft und die Positionierung zu Inhalten aus dem Fokus gerieten. Doch gerade mit Blick auf die oben formulierten Merkmale und die Betonung einer intendierten Förderung des öffentlichen Wohls lässt sich angesichts einer zunehmenden Reduktion der Debatte auf Fragen der Wissenschaftskommunikation vielmehr von einer, Soziologie in der Öffentlichkeit' als von einer ,öffentlichen Soziologie` sprechen - was nicht zuletzt als unmittelbare Folge von digitalisierter Wissenschaftskommunikation gesehen wird (Healy 2017). Dadurch besteht die Gefahr, dass das Verständnis der Problemhorizonte (Bude 2005: 378) verloren geht. Entsprechend macht Bude (ebd.) deutlich: „Wer mit der Gesellschaft in Kontakt treten will, muss über ein Verständnis der ihr eigenen Problematisierungshorizonte verfügen. Sonst befriedigt man in erster Linie sich selbst, verpasst aber die Leute.“ Möchte die Disziplin allerdings als eine für die gesellschaftliche Gestaltung wichtige Kraft wahrgenommen werden, ist neben der Aufbereitung von Inhalten und der Diskussion von Formaten unserer Meinung nach ein soziologischer Dreiklang notwendig, der unter Rückgriff auf unterschiedliche Klassiker mit den Begriffen der Normativität, Totalität und Rückbindung beschrieben werden kann. Basierend auf den Überlegungen $\mathrm{zu}$ neuen Formen der Wissenschaftskommunikation werden also zunehmend auch Podcast-Formate entwickelt, um als Disziplin in der Öffentlichkeit Gehör zu finden. Den Anspruch kritisch zu sein erheben - analog zu einem vielfach geäußerten, eher allgemeinen Selbstverständnis in der Soziologie (Lessenich 2014; Vobruba 2013) - vermutlich alle. Doch wann und inwiefern kann von einem interventionistischen Charakter des Formats gesprochen werden?

\subsection{Pragmatische Gedanken zur gesellschaftskriti- schen Fundierung}

Dass eine kritische Soziologie von Bedeutung ist, hebt Lessenich (2014: 13) hervor und betont, es gebe ",gute empirische Gründe dafür, die Sozialkritik wieder in den Mittelpunkt soziologischer Praxis zu rücken. Und die empirischen Gründe dafür sind deswegen besonders stark, weil die Rückkehr der Sozialkritik in die Soziologie zwar ganz offensichtlich durch die Krise bzw. multiplen Krisen der jüngsten Zeit angetrieben wurde, die soziale Realität aber, auf die sich diese Kritik bezieht, alles andere als konjunktureller Natur ist“. Es stellt sich somit die Frage, was eine kritische Soziologie als Basis möglicher Interventionen ausmacht.

Soziologien, die diese Intervention in ihren Ansätzen grundsätzlich mittragen, sind neben den frühen Vertretern der Kritischen Theorie auch die Arbeiten von Charles W. Mills, der in der Debatte zur „Public Sociology“ ebenfalls eine wesentliche Rolle spielt. Um nützliche Hinweise zu dezidiert gesellschaftskritischem Vorgehen zu bekommen, welches sich von partieller Kritik bestimmter Sachverhalte abhebt und möglichst umfassend ist, soll im Folgenden Mills (1959/2016) Idee der „soziologischen Phantasie“ in Beziehung zu ergänzenden Postulaten der Kritischen Theorie gesetzt werden. Sein Verhältnis zur Kritischen Theorie sieht Mills als ein Wechselspiel von Nähe und Distanz (Neun 2019: 93). Wir wollen uns im Folgenden auf Aspekte konzentrieren, die wir als fruchtbar im Sinne unseres Vorhabens empfinden. Drei Gemeinsamkeiten fallen hierbei besonders auf: Mit der Verwendung psychologischer Begriffe (1) zur Charakterisierung der gegenwärtigen Gesellschaft schließt Mills nicht zuletzt an den Charakterbegriff von Fromm an. Auch weist er - ganz im Sinne der Kritischen Theorie (Horkheimer 1931/2009) - sowohl allzu umfassende Großtheorie oder abgehobene Metaphysik als auch abstrakten Empirismus zurück (2): Wer das falsche Ganze analysieren und kritisieren will, darf sich demnach weder durch $\mathrm{zu}$ metaphysische Abstraktion noch durch (absichtlich) die Sicht verstellenden Konkretismus leiten lassen. So lässt sich sein Werk - wie jenes der Kritischen Theorie - als „Projekt einer sozialwissenschaftlichen Zeitdiagnose in emanzipatorischer Absicht" (Dubiel 2001: 22) begreifen.

Zudem greift Mills in seinem Verständnis sozialwissenschaftlicher Forschung auch eine Differenzierung Horkheimers (1937/2011) auf (3): Mit diesem kann - etwas holzschnittartig - zwischen traditioneller und kritischer Theorie unterschieden werden. Die traditionelle Vorstellung von Theorie ergibt sich dabei durch die bestehende gesellschaftliche Arbeitsteilung, innerhalb derer Wissenschaft ein gesellschaftlicher Teilbereich ohne (direkten) Bezug zu anderen gesellschaftlichen Teilbereichen ist (ebd.: 214). Ins Zentrum der Differenzierung traditioneller und kritischer 
Wissenschaftskonzeptionen wollen wir daher nicht zuletzt die Begriffe der Normativität, Totalität und Rückbindung stellen. Während bei der Entwicklung der traditionellen Theorie „das wissenschaftliche Denken [...] diese Einheit von Werturteil und Analyse zerbrechen [musste]“ (Marcuse 1967/2014: 142), ist es erstens notwendig, Normativität in der Wissenschaft anzuerkennen und $\mathrm{zu}$ erlauben auch darüber nachzudenken, was sein sollte im Gegensatz zu dem, was ist. Neben der - für die traditionelle Theorie geradezu konstitutiven - Beschneidung der kritischen Perspektive ist deren Besonderung ein weiterer Mechanismus, der sie zu einem Randphänomen gesellschaftlicher Problemdiagnose macht. Kritische Theorien und Konzepte kämpfen daher nicht nur um ihre bloße Existenz, sondern auch darum, im Diskurs als sagbare wie relevante Perspektive wahrgenommen zu werden: „Die Möglichkeit einer gänzlich anderen gesellschaftlichen Organisation des Lebens hat nichts mit der ,Möglichkeit' gemein, daß morgen an allen Türeingängen ein Mann mit einem grünen Hut erscheint; aber ihre Behandlung nach derselben Logik kann dazu dienen, unerwünschte Möglichkeiten zu diffamieren“ (Marcuse 1967/2014: 228).

Die Anerkennung der Normativität in der Wissenschaft bedeutet somit nicht nur, normative Beiträge zu ermöglichen, sondern auch, diese gegen allzu vorschnelle Fundamentalkritik ebenso zu verteidigen wie gegen das Abschieben in die (moralinsaure und wenig ergiebige) ,Welt der Werte‘. Anders formuliert gilt es, die Möglichkeit kritischer Normativität gegen die - zumeist implizite - bürgerliche Normativität des Faktischen (Horkheimer 1937/2011: 216) in Stellung zu bringen - und das jenseits einer Welt der noblen Werte, da diese als subjektiv und chancenlos gegen die Kraft der Rationalität gilt (Marcuse 1967/2014: 162). Eine solche Form kritischen, normativen Denkens kann auch als eine Voraussetzung zur (Weiter-)Entwicklung einer Gesellschaft jenseits der Pfade vorgeblicher Alternativlosigkeiten angesehen werden (Horkheimer 1937/2011: 225) und als eine Kritik an einem verkürzten Rationalitätsbegriff, der diese auf (vorgebliche) ökonomische Nützlichkeit verkürzt (Drengson 2005).

Zweitens ist für ein solches kritisches Verhalten eine Perspektive notwendig, wie sie Horkheimer in seiner zentralen Frage für die Sozialforschung formuliert: „Welche Zusammenhänge lassen sich bei einer bestimmten gesellschaftlichen Gruppe, in einer bestimmten Zeitspanne, in bestimmten Ländern nachweisen zwischen der Rolle dieser Gruppe im Wirt- schaftsprozeß, der Veränderung in der psychischen Struktur ihrer einzelnen Mitglieder und den auf sie als Gesamtheit im Ganzen der Gesellschaft wirkenden und von ihr hervorgebrachten Gedanken und Einrichtungen“ (Horkheimer 1931/2009: 33). Zentral ist also, eine ganzheitliche Perspektive zu etablieren, die die Totalität der Gesellschaft mitdenken muss und sich nicht auf Einzelaspekte konzentriert (Horkheimer 1937/2011: 223f.) bzw. sogar versucht diese zu isolieren (ebd.: 216). Die Frage nach Totalität oder Vereinzelung ist mitnichten bloß eine der Perspektive auf die soziale Welt, beispielsweise die Differenz von Mikro- und Makroebene. Vielmehr erscheinen dem kritischen Denken Missstände und Problemstellungen als „notwendig mit der ganzen Einrichtung des Gesellschaftsbaus verknüpft “ (ebd.: 223).

Im Gegensatz hierzu verändern die Gewohnheit eines isolierenden Empirismus wie auch die vereinzelnde Produktionsweise den affirmativen Blick auf Gesellschaft (ebd.: 218). Das Pochen auf Totalität entzieht sich somit dezidiert dem Schielen auf - wenn auch meist unbewusst, so doch immer wieder aktiv zu isolierende Einzelphänomene.

Drittens handelt das kritische Denken jedoch nicht von einer ursprünglichen Wahrheit oder Sinn und Wirklichkeit jenseits von Geschichte und Gesellschaft (Schwandt 2010). Kritisches Denken ist nicht ahistorisch, vielmehr ist es ein konstitutives Merkmal bereits der ersten Generation der Kritischen Theorie, die Verbindung von Abstraktion (bzw. im obigen Duktus: Totalität) und Rückbindung an gesellschaftliche und letztlich individuelle Problemlagen. Oder, wie Mills es in seiner Arbeit zur soziologischen Phantasie ausdrückt: „Bei unserer Auswahl und Formulierung der Probleme müssen wir, erstens, Gleichgültigkeit in ein öffentliches Problem, übersetzen ' und Unbehagen in ein privates, und dann, zweitens, beide Arten von Problemen in unsere Fragestellung aufnehmen. In beiden Schritten müssen wir die beteiligten Werte und ihre Gefährdungen so einfach und genau wie möglich darlegen und versuchen, sie miteinander zu verknüpfen“ (Mills 1959/2016: 199). Denn individuelle Erlebnisse zu begreifen gelingt gemäß Mills nur dann, wenn das eigene Schicksal in die Entwicklungen der eigenen Schicht und zeitgenössischer Dynamiken eingeordnet werden kann. Demnach, so fasst es Lessenich in seinem Vorwort zur deutschen Neuausgabe von Mills Werk zusammen, sei die Aufgabe von Soziolog*innen „in ihrer wissenschaftlichen Forschung und ihrer öffentlichen Kommunikation ,Mensch und Gesellschaft, 
Tabelle 1: Vier Felder soziologischer Kommunikation

\begin{tabular}{lll}
\hline & Alltagsweltlich-konnotierte Diskurse & Wissenschaftlich-elaborierte Diskurse \\
\hline Soziologie in der Öffentlichkeit & Einführend-grundlegend & Theoretisch-konzeptionell \\
Öffentliche Soziologie & Zeitdiagnostisch-appellativ & Theoretisch-K/kritisch \\
\hline
\end{tabular}

Quelle: Eigene Darstellung

Biographie und Geschichte, Selbst und Welt' zueinander in Beziehung zu setzen. Soziologische Phantasie vermag aufzuzeigen, dass ,private Schwierigkeiten immer auch Ausdruck ,öffentlicher Probleme sind“ (Lessenich 2016: 14). Während private Schwierigkeiten sich auf den Charakter und die sozialen Beziehungen des Einzelnen und dessen begrenzten sozialen Lebensbereich beziehen, betreffen öffentliche Probleme die über lokale Umwelten des Individuums hinausgehenden Strukturen (Mills 1959/2016). Letztlich macht es diesen Dreiklang von Normativität, Totalität und Rückbindung aus, der eine Entwicklung der Soziologie zur „methodisch versierten Reflexionsinstanz“ (Lamla 2014) und damit zu einer Basis für gesellschaftliche Interventionen unterstützen kann. Kritische Theorie fungiert so als „Diagnose sozialer Pathologien und Emanzipation als Entwicklungsverlauf von der Krankheit zur Genesung"(Allen 2019: 428).

\subsection{Vier Felder soziologischer Kommunikation in und mit der Öffentlichkeit}

Dass sich die Soziologie mit Formen der Wissenschaftskommunikation und dem Erreichen der Öffentlichkeit auseinandersetzt, scheint hinreichend offensichtlich zu sein. Doch wird nicht grundsätzlich dem gleichen Modus gefolgt, sodass eine Einordnung und Analyse soziologischen Wirkens in der Öffentlichkeit sinnvoll erscheinen. Wie bereits skizziert, plädieren wir in dieser Frage zum einen für eine „(E-)Public Sociology“, die mehr ihren Inhalt und dessen Kommunikation in den Mittelpunkt stellt und weniger das jeweilige Medium, welches nach unserer Lesart lediglich ein (austauschbares) Mittel zum Zweck ist. Gerade mit Blick auf interventionistisches Potenzial ist dies, wie im voranstehenden Kapitel erläutert, über das Zusammendenken der wissenschaftstheoretischen Positionen von Kritischer Theorie und Mills möglich, um so die tiefergehende Integration der Perspektiven zu ermöglichen und (insbesondere über Mills) an die bisherigen Diskurse zur „Public Sociology“ anzuschließen. Zum anderen nutzen wir diese wissenschaftstheoretischen Positionen und Zielsetzungen aus der Kritischen
Theorie, um den Möglichkeitsraum für soziologische Interventionen (oder deren Versuch) konzeptionell zu erweitern. Aufbauend darauf unterteilen wir die Beziehung der Soziologie zur Gesellschaft idealtypisch in vier Typen bzw. Felder, je nach Inhalt und diskursiven Anknüpfungspunkten.

Inhaltlich differenzieren wir hierbei zwischen einer ,Soziologie in der Öffentlichkeit ‘ und einer ,öffentlichen Soziologie‘ im Sinne Burawoys (2005). Auch wenn nur einer der beiden Typen die von Burawoy verwendete Bezeichnung trägt, trifft auf beide das erste von Neun (2018) im Anschluss an Brady (2004) definierte Kriterium zu (s. o.), da auch die Soziologie in der Öffentlichkeit versucht, ein breites Publikum zu erreichen. Den zentralen Unterschied stellt demnach das zweite Kriterium dar, namentlich der Versuch, „das öffentliche Wohl zu fördern“ (Neun 2018: 14) und damit in bestehende gesellschaftliche Prozesse zu intervenieren. Anders formuliert: Das Ziel der Soziologie in der Öffentlichkeit liegt darin, ein (potenziell) breites Publikum mit soziologischem Denken sowie soziologischen Konzepten und Theorien vertraut zu machen. Dabei bleibt zunächst offen, ob diese auf ein spezifisches gesellschaftliches Problem bezogen oder ihre Zielsetzung in der Skizze eines konzeptionellen/theoretischen Ansatzes, einer Denkschule oder dem Cuvre einer Soziologin oder eines Soziologen verortet werden. Gesetzt ist dagegen, dass die entsprechenden Arbeiten sich jenseits oder neben (sonstiger) gesellschaftlicher Wirklichkeit befinden und nicht in diese eingreifen wollen - von der rein intellektuellen Ebene der Analyse abgesehen.

Öffentliche Soziologie dagegen kann im Sinne der Kritischen Theorie (s. o.) und Mills' als normativ gelten. Hier wird - explizit oder implizit - vor dem Hintergrund einer Differenz zwischen Wirklichkeit und Begriff, also zwischen einer empirisch vorgefundenen Welt und begrifflichen Potenzialen, argumentiert, wobei die beiden Typen der öffentlichen Soziologie wie zu zeigen sein wird - hierbei sehr unterschiedlich vorgehen.

Dies verweist auf die zweite, die horizontale Achse. Während die vertikale auf inhaltliche Aspekte und insbesondere auf die Frage nach Normativität verweist, 
steht bei der horizontalen die Art der Kommunikation und der Anschluss an einen alltagsweltlich-konnotierten oder einen wissenschaftlich-elaborierten Diskurs im Fokus. Ersterer bedeutet den Versuch, soziologisches Denken möglichst zugänglich darzustellen und in alltagsweltlichem Vokabular, mit ,gebräuchlichen Begrifflichkeiten auszudrücken. Letzterer dagegen versucht, ein (auch) fachfremdes Publikum ,mitzunehmen in ein an (sub-)fachlichen und (sub-)konzeptionellen Gegebenheiten orientiertes Schreiben und Darstellen. Für die vier durch die beiden Achsen aufgespannten Idealtypen bedeutet dies im Einzelnen:

- Einführend-grundlegender Typus: Ziel der Veröffentlichungen, die in diesem Typus zusammengefasst werden, ist die Skizze soziologischen Denkens, soziologischer Denkschulen, Theoriegebäude oder einzelner Soziolog*innen in einer allgemeinverständlichen Sprache mit erklärender Funktion. Soziologie wird so themen- oder konzeptzentriert vermittelt und ein solches ,Näherbringen 'soziologischer Theorien oder soziologischen Denkens ist das (eigentliche) Ziel der hier versammelten Veröffentlichungen. Das Grundkonzept des Vorgehens erinnert an Lehrbücher, ist in der Regel allerdings weniger umfassend, weniger schematisch und tendenziell eher an allgemeine Anschlussfähigkeit hin angepasst, avisierte Konsument*innen sind nicht Studierende, sondern Interessierte (die natürlich auch Studierende sein können). Blickt man auf die Printlandschaft, wäre an dieser Stelle die „10 Minuten Soziologie“-Reihe des Transcript Verlages zu nennen. Beispiele für diesen Typus im Bereich von Podcasts im deutschen Sprachraum sind der vielfach ausgezeichnete „Soziopod“", der in einem dialogischen Format grundlegende Autor*innen aus der Soziologie erläutert, oder „Soziologie on air “" 4 der in der „Länge einer U-Bahnfahrt [...] Einblicke in die Welt der Soziologie“ verspricht und dabei „so sparsam wie möglich mit dicken Theoriebrettern“ sein möchte. Im internationalen Kontext ist etwa der Podcast „The sociology show ${ }^{\text {“5 }}$ zu nennen, in dem Interviews mit unterschiedlichen Personen aus dem Bereich der Soziologie geführt werden.

- Theoretisch-konzeptioneller Typus: Ziel der Veröffentlichungen, die in diesem Typus zusammengefasst werden, ist das Darlegen soziologischen Denkens

3 https://soziopod.de [letzter Zugriff: o8.06.2021].

4 https://soziologieonair.de

[letzter Zugriff: o8.06.2021].

5 https://podcasts.apple.com/gb/podcast/the-sociology-show/id1507196347 [letzter Zugriff: 08.06.2021]. und soziologischer Theorien wie Konzepte in einer tendenziell wissenschaftlich-elaborierten Sprache mit der Funktion, sowohl das Alltagsdenken als auch das soziologische Denken zu inspirieren und weiterzuentwickeln. Das Ziel einer gewissen Anschlussfähigkeit an alltagsweltliches Denken, beispielsweise durch die Beschäftigung mit einer relevanten gesellschaftlichen oder gesellschaftspolitischen Fragestellung, wird mit dem Fokus auf wissenschaftlichen Fortschritt verbunden. Das Grundkonzept des Vorgehens erinnert an theoretische wie empirische Veröffentlichungen aus dem Fachbereich, allerdings ist eine breitere Anschlussfähigkeit über soziologisches Fachpublikum hinaus intendiert. Veröffentlichungen aus diesem Bereich können direkten Bezug zu aktuellen, gesellschaftlich relevanten Themenstellungen aufweisen, beschränken sich jedoch dezidiert auf die analytische Ebene und wollen keinen Einfluss nehmen - vom Anregen der intellektuellen Auseinandersetzung mit dem Thema abgesehen. Ein Beispiel für Veröffentlichungen dieses Typus im Printbereich kann das Werk „Das große Nein. Eigendynamik und Tragik des gesellschaftlichen Protests“ (2020) von Armin Nassehi sein: Der Autor versucht an vielen Stellen, auch soziologisch ungeübte Leser*innen ,mitzunehmen', dennoch ist der Text zum Großteil in wissenschaftlich-elaborierter Sprache gehalten und grenzt sich somit implizit vom einführend-grundlegenden Typus ab. Die Abgrenzung zur Ebene der öffentlichen Soziologie erfolgt explizit und hält laut eigener Aussage „einen ziemlich weiten Sicherheitsabstand zu Protestkommunikation“ (ebd.: 6). Podcasts, die diesem Typus zugerechnet werden können, sind im deutschen Sprachraum etwa "Andreas Reckwitz im Gespräch"6 in dem der Soziologieprofessor Andreas Reckwitz sein Buch „Die Gesellschaft der Singularitäten“ (2019) bespricht, sowie „sozialtheoristen “7 in deren Podcast „das sprechende Denken" in den Mittelpunkt gestellt und mehrere innersoziologische Diskurse thematisiert werden. Aber auch der Podcast „Soziologische Perspektiven auf die Corona-Krise ${ }^{\text {“8 }}$ des Wissenschaftszentrums Berlin für Sozialforschung (WZB) kann hier genannt werden.

- Zeitdiagnostisch-appellativer Typus: Ziel der Veröffentlichungen, die unter diesem Typus dargelegt werden, ist das zugespitzte Aufzeigen gesellschaftlicher

6 https://andreasreckwitz.podigee.io [letzter Zugriff: 08.06.2021].

7 https://sozialtheoristen.de/category/podcast/ [letzter Zugriff: 08.06.2021].

8 https://coronasoziologie.blog.wzb.eu [letzter Zugriff: 08.06.2021]. 
Problemstellungen verbunden mit einem - zumindest impliziten - Apell, an diesen Problemstellungen etwas zu verändern bzw. sie zum Ausgangspunkt von Gegenwehr zu machen. Hierzu wird ein Thema oder ein Themenbündel mit aktueller, gesellschaftspolitischer Relevanz herausgegriffen, die große Bedeutung der Themenstellung wird aufgezeigt und (mindestens implizit) wird die Notwendigkeit von Gegenmaßnahmen postuliert. Die Sprache schließt an den alltagsweltlichen Sprachduktus an, Fachausdrücke werden erklärt und die Texte sind nicht selten appellativ gehalten, wobei die Grenzen zur Skandalisierung fließend sind. Als ,Klassiker' dieses Typus im Printbereich können die Veröffentlichungen von Ulrich Beck über die „Risikogesellschaft" (1986), die "Schöne neue Arbeitswelt“ (1999) und - allgemeiner gefasst - die Reflexive Moderne gelten. In den vergangenen Jahren gab es darüber hinaus steigende Veröffentlichungszahlen beispielsweise von Bude (2013; 2014), Nachtwey (2016) und Streeck (2015). Letzterer kann hier als Gewährsmann für unsere Konzeption dieses Typus stehen, arbeitet er doch mustergültig die Dringlichkeit der von ihm skizzierten Problemstellung der "Gekauften Zeit“ und des Lebens in einer Schuldner-Gesellschaft, in der das Kapital durch den hohen Kreditbedarf des Staatswesens immer mehr Macht als Gläubiger (neben jener als Dienstherr der Bürgerinnen) akkumuliert, auf. Im nationalen Kontext findet sich etwa der „Dissens“Podcast, der laut eigener Aussage thematisiert, „was in unserer Gesellschaft falsch läuft. Denn: Kritisieren, was ist, heißt sagen, was geändert werden muss. ${ }^{\text {“9 }}$ Es wird damit explizit auf die Notwendigkeit von Veränderung Bezug genommen. Außerdem bietet auch der Podcast „Das neue Berlin“ ein Beispiel für diesen Typus. In diesem Podcast ist der gesellschaftliche Veränderungswunsch etwas impliziter, dennoch werden Themen des aktuellen gesellschaftlichen Problemhorizonts aufgegriffen und versucht, „Perspektiven aus den Geistesund Sozialwissenschaften zeitdiagnostisch fruchtbar zu machen "10.

- Theoretisch-K/kritischer Typus: Ziel der Veröffentlichungen, die unter diesem Typus subsummiert werden, ist eine explizit normative, zumeist an das Erleben von Gesellschaftsmitgliedern rückgebundene und - im Gegensatz zum zeitdiagnostisch-

9 https://podcast.dissenspodcast.de [letzter Zugriff: 08.06.2021].

10 https://dasneue.berlin/ueber-uns/ [letzter Zugriff: 08.06.2021] appellativen Typus - die gesellschaftliche Totalität betonende Darstellung, die sprachlich als wissenschaftlich-elaboriert zu skizzieren ist. Der zentrale Unterschied zum zeitdiagnostisch-appellativen Typus ist - neben den Differenzen in der Kommunikation der Fokus auf einzelne Phänomene oder gesellschaftliche Totalität: Während der zeitdiagnostisch-appellative Typus einzelne Themen oder Themenbündel fokussiert und deren Relevanz für wesentliche gesellschaftliche Entwicklungen postuliert, stellen die theoretisch-K/ kritischen Ansätze gerade die gesellschaftliche Totalität und die Zusammenhänge wie Wechselwirkungen zwischen verschiedenen gesellschaftlichen Teilbereichen dar. Daraus ergeben sich auch Unterschiede in Bezug auf die anderen beiden genannten Aspekte: Die Normativität ist eher eine grundlegende Haltung statt eines Plädoyers für oder gegen einen Aspekt der sozialen Welt und der Rückbezug auf das Leben von Gesellschaftsmitgliedern ist eher abstrakt (oder vermag zumindest so zu erscheinen). Als ,Klassiker dieses Typus im Printbereich, die Namensgeber einer der beiden Schreibweisen sind, kann die Kritische Theorie gelten, als aktueller Vertreter beispielsweise Hartmut Rosa mit seinem Werk „Resonanz“ (2016) oder - im internationalen Kontext und mit einigen Jahren Abstand - die Gesellschaftsdiagnosen von Pierre Bourdieu (u. a. 1998) oder Luc Boltanski (2010). Als Podcast kann hier „Wohlstand für alle ${ }^{\text {“ll }}$ genannt werden: Die beiden Protagonisten setzen sich mit zumeist dezidierten - Bezügen zur Kritischen Theorie und (vor allem) zum Marxismus mit aktuellen gesellschaftspolitischen Themenstellungen auseinander und kritisieren auch die Influencer-Szene als neue Vorhut der Produktion künstlicher Bedürfnisse so scharf wie fundiert (Nymoen/Schmitt 2021). Aber auch der Podcast „future histories“12 kann darunter gefasst werden. So weisen schon Titel und Untertitel (,zur Erweiterung unserer Vorstellung von Zukunft“) auf ein prozesshaftes Verständnis gesamtgesellschaftlicher Entwicklung sowie des Anspruchs auf ein gewisses $\mathrm{Maß}$ an Gestaltbarkeit von Zukunft hin. Wobei dies offen und in Dialog mit der Öffentlichkeit passieren solle.

Wichtig zu betonen ist uns, dass wir die Einteilung in diese vier Typen dezidiert nicht als wertend verstan-

\footnotetext{
11 https://wohlstandfueralle.podigee.io [letzter Zugriff: o8.06.2021].

12 https://www.futurehistories.today [letzter Zugriff: o8.06.2021].
} 
den wissen wollen. Beispielsweise ist Alltagssprache nicht per se besser als wissenschaftlich-elaborierte oder umgekehrt. Wohl aber fällt uns auf, dass insbesondere der Theoretisch-K/kritische Typus von tendenziell abnehmender Provenienz ist und insbesondere in der „E-Public Sociology“ nur eine sehr untergeordnete Rolle spielt - eine Entwicklung, die wir problematisieren möchten. Insbesondere die ständig verfügbaren und auf Schnelligkeit wie schnelle Konsumierbarkeit setzenden Medien wie Podcasts bevorzugen - so unsere These - alltagsweltlich gehaltene Formate, die sich politisch zwischen unpolitischem Gebaren und thematischer Einseitigkeit positionieren. Dies muss kein Problem darstellen und kann sowohl in das soziologische Denken einführen wie auch den Fokus auf gesellschaftliche Problemstellungen scharf stellen. Es könnte jedoch ebenso zu einer Profanisierung des Denkens über Soziologie und soziale Erregungsspiralen beitragen.

\section{Diskussion}

Doch was bedeutet dies nun für die Frage nach dem interventionistischen Potenzial von Podcasts zu soziologischen Themen? Unabhängig von persönlichen Vorlieben und Urteilen über „Public Sociology“ und soziologische Zeitdiagnosen plädieren wir für eine gewisse Anerkennung dieser Lesarten, „da mit dem Konzept öffentliche Soziologie etwas im Raum steht, das mehr und anderes andeutet als die Modernisierung unserer Disziplin durch eine auszubauende PR-Abteilung“ (Damnitz 2013: 252). So weist auch Mills (1960) darauf hin, dass die Lektüre klassischer soziologischer Texte schon zu der Zeit ihrer Veröffentlichung über die reine Informationsbeschaffung hinausging. So gehe es vielmehr darum, Orientierung aus diesen $\mathrm{zu}$ ziehen. Die Beschäftigung mit Soziologie ist - zumindest außerhalb reiner Fachdiskussionen - somit selten eine nur um der soziologischen Erkenntnis willen. Zur - wie gezeigt - unabgeschlossenen Diskussion um den Stellenwert von „Public Sociology“ und soziologischer Zeitdiagnosen kommen mit der „E-Public Sociology" und Ansätzen wie dem Podcasten neue Herausforderungen und eine Ausdifferenzierung der Modi von Wissenschaftskommunikation hinzu. Mit Blick auf Podcasts stellten wir im Text die Frage nach Kriterien, mittels derer soziologische Podcasts als ,legitime' gesellschaftliche Intervention gelten können. Mit der Differenzierung von ,öffentlicher Soziologie und ,Soziologie in der Öffentlichkeit` argumentieren wir - in Übereinstimmung mit einer neu verstandenen Kritischen Theorie (Dörre et al. 2009; Neun 2019) -, dass als Antwort auf das „Einflussdefizit der Disziplin“ (Lamla 2014: 493) Systematischeres gefordert ist als die bloße Steigerung der „Präsenz der Soziologie“ (Treibel/ Selke 2012: 417). Gerade vor dem Hintergrund einer mit Blick auf derzeitige gesellschaftliche Entwicklungen von Heitmeyer et al. (2020: 291f.) diagnostizierten „entsicherten Unübersichtlichkeit“ scheinen diese systematischen Angebote auch von soziologischer Seite als zentral, um sich bei der gesellschaftspolitisch zentralen Frage einzubringen, „wie sich die offene Gesellschaft und die liberale Demokratie weiterentwickeln können“" und sich nicht lediglich als eine Art „technologische Gesellschaftsverwaltung “ zu betätigen. Anzumerken ist dabei jedoch auch, dass in einem solchen - Werturteile umfassenden - Verständnis der Disziplin „eine Wechselbeziehung von empirischer Recherche und wissenschaftlich begründeter Sozialkritik angelegt [ist], die sich zwingend an den Standards zu orientieren hat, wie sie seitens der professionellen Soziologie definiert werden“ (Aulenbacher et al. 2017a: 24).

Doch auch wenn man insbesondere an dem immer umfangreicher werdenden Angebot an Podcasts mit soziologischem Hintergrund (ein Trend, der sich mit der Pandemie verstärkt zu haben scheint), die zunehmende Beschäftigung der Disziplin mit Formen der Wissenschaftskommunikation und das Erreichen der Öffentlichkeit konstatieren kann, lässt sich fragen, ob und inwiefern dies zu einer erhöhten Relevanz im Kontext öffentlicher wie politischer Diskurse und damit der Möglichkeit von Intervention beiträgt.

Dies ist nicht zuletzt deshalb offen, weil auch beim soziologischen Podcast nicht einem grundsätzlich gleichen Modus des Adressierens der Öffentlichkeit gefolgt wird. Um unter den Umständen veränderter Voraussetzungen bei nach wie vor bestehendem Einflussdefizit der Soziologie urteilsfähig zu bleiben, schlagen wir mit dem vorliegenden Beitrag vier Idealtypen verschiedener soziologischer Adressierungen der Öffentlichkeit vor. Für diese liegen beim Podcasten im Grunde gute strukturelle Voraussetzungen vor: „Sie [die Form des Podcasts] muss sich nicht den Aufmerksamkeits- und Verwertungslogiken professioneller Medien unterwerfen und ist dennoch erreichbar für all jene, die das Thema wirklich interessiert" (Schwarz/Wetzel 2019: 82). Zudem wird über das Sprachliche eine gewisse Nähe und Vertrauenswürdigkeit geschaffen. Das heißt, Podcasten erreicht nominell auch Personengruppen, die sich jenseits von öffentlichen, zumeist bildungsbür- 
gerlich geprägten Diskursen im Feuilleton bewegen. Die Genese eines Verständnisses für soziologische Problemhorizonte scheint damit auch bei ,neuen' Personengruppen möglich zu sein. Möchte man allerdings den Podcast als „Feuilleton von unten“ ernst nehmen und die „E-Public Sociology“ als kritische soziologische Intervention denken, kann man an dieser Stelle nicht stehen bleiben, wenn es darum geht, wie Soziologie ihr (kritisches) Potenzial mit einer gewissen Konkretion und ohne (zu) viel außer-soziologischem Ballast nutzen kann.

Und genau in diesem Punkt zeigen die von uns identifizierten Typen ganz grundsätzliche Unterschiede. Denn gerade die beiden Formen des ,einführendgrundlegenden Typs und des ,theoretisch-konzeptionellen Typs' verbleiben bei eher beschreibenden Sachverhalten, bei denen zwar oftmals äußerst gekonnt an die Öffentlichkeit herangetreten wird, die Problemdiagnose und damit der Hebel und die Einsicht in Notwendigkeiten potenzieller Intervention bleiben in der Regel jedoch aus. Kritisch betrachtet erfolgt über die abgetrennte Betrachtung von Theorien, Personen oder Einzelphänomenen eine gesellschaftlich-affirmative Situation. Das Potenzial zur kritisch-interventionistischen Haltung des Podcasts bleibt unausgeschöpft.

Die Typen der ,zeitdiagnostisch-appellativen` und ,theoretisch-K/kritischen' Kommunikation räumen der Veränderung eine zentrale Rolle ein und können im Gegensatz zu der immanent verbleibenden Kritik der beiden anderen Typen als interventionistisch verstanden werden. Bei diesen Typen wird das gesellschaftspolitische Problembewusstsein - oftmals unter Rückbindung an gesellschaftlich betroffene Akteur*innen - herausgestellt. Mit dem normativen Anspruch geschieht die Benennung und Herausarbeitung gesellschaftlicher Problemstellungen nicht nur deskriptiv-analytisch, sondern analytisch-radikal, da mit den Gästen oftmals bereits Interventionspunkte dargelegt werden. Gerade bei dem Typus des ,theoretisch-K/kritischen' geschieht dies unter Bezugnahme auf die historisch konkreten Bedingungen der Phänomene. Dabei gelten uns die den beiden ersten Typen zugeordneten Podcasts nicht als weniger gut diese sind alle äußerst informativ und teils werden hoch komplexe Themen in gekonnter Manier transportiert -, aber mit Blick auf Kriterien der Intervention (ein Anspruch, der von den jeweiligen Podcasts vielleicht gar nicht gehegt wird), muss gesagt werden, dass dieses Potenzial des Podcasts dort nicht ausgeschöpft wird.

Wir plädieren für eine Offenheit allen Typen gegenüber - und somit ganz dezidiert auch für einen Typus, der aufgrund seiner Explikation von Normativität und Komplexität der Betrachtung auf den ersten Blick als wenig anschlussfähig erscheinen mag. Auf diese Art nutzen wir aus der Kritischen Theorie entwickelte Positionen, um den Möglichkeitsraum für soziologische Interventionen (oder deren Versuch) konzeptionell zu erweitern. Das bedeutet, dass auch Podcasten mit einem Geltungsanspruch im Sinne öffentlicher Soziologie impliziert, diese Kriterien in die Kommunikation soziologischer Forschung integrieren und im Sinne einer Intervention umsetzen zu können. Dabei heben wir hervor, dass eine „Public Sociology“ notwendig ist, die mehr ihren Inhalt und dessen Kommunikation in den Mittelpunkt stellt und weniger das jeweilige Medium, welches nach unserer Lesart lediglich ein (austauschbares) Mittel zum Zweck ist. In der Konsequenz heben wir den Dreiklang von Normativität, Totalität und Rückbindung heraus, der die Etablierung der Soziologie zur „methodisch versierten Reflexionsinstanz" (Lamla 2014: 497) unterstützen kann.

\section{Literatur}

Allen, A. (2019): Psychoanalyse, Kritik und Emanzipation. In: Bohmann, U./Sörensen, P. (Hg.): Kritische Theorie der Politik. Frankfurt/M.: Suhrkamp, 426-449.

Aulenbacher, B./Dörre, K./Burawoy, M./Sittel, J. (2017a): Zur Einführung: Soziologie und Öffentlichkeit im Krisendiskurs. In: Aulenbacher, B./Dörre, K./Burawoy, M./Sittel, J. (Hg.): Öffentliche Soziologie: Wissenschaft im Dialog mit der Gesellschaft. Frankfurt/M.: Campus Verlag, 11-30.

Aulenbacher, B./Dörre, K./Burawoy, M./Sittel, J. (2017b): Öffentliche Soziologie: Wissenschaft im Dialog mit der Gesellschaft. Frankfurt/M.: Campus Verlag.

Beck, U. (1986): Risikogesellschaft. Frankfurt/M.: Suhrkamp.

Beck, U. (1999): Schöne neue Arbeitswelt. Frankfurt/M.: Suhrkamp.

Böhle, F./Bolte, A./Huchler, N./Neumer, J./Porschen-Hueck, S./Sauer, S. (2014): Vertrauen und Vertrauenswürdigkeit. Arbeitsgestaltung und Arbeitspolitik jenseits formeller Regulierung. Wiesbaden: Springer VS.

Boltanski, L. (2010): Soziologie und Sozialkritik. Berlin: Suhrkamp.

Bourdieu, P. (1998): Gegenfeuer: Wortmeldungen im Dienste des Widerstands gegen die neoliberale Invasion. Konstanz: Universitätsverlag Konstanz.

Brady, D. (2004): Why Public Sociology May Fail. Social Forces, 82 (4), 1629-1638.

Bude, H. (2005): Auf der Suche nach einer öffentlichen Soziologie: Ein Kommentar zu Michael Burawoy von Heinz Bude. Soziale Welt, 56 (4), 375-380. 
Bude, H. (2013): Bildungspanik: Was unsere Gesellschaft spaltet. München: dtv.

Bude, H. (2014): Gesellschaft der Angst. Hamburg: Hamburger Edition.

Burawoy, M. (2005): For public sociology. Soziale Welt, 56 (4), 417-439.

Burawoy, M. (2015): Public Sociology: öffentliche Soziologie gegen Marktfundamentalismus und globale Ungleichheit. Weinheim Basel: Beltz Juventa.

Damitz, R. M. (2013): Soziologie, öffentliche. Soziologische Revue, 36 (3), 251-62.

Dörre, K./Lessenich, S./Rosa, H. (2009): Soziologie - Kapitalismus - Kritik: eine Debatte. Frankfurt/M.: Suhrkamp.

Drengson, A. (2005): Self-Realization: An Ecological Approach to Being in the World. The Selected Works of Arne Naess. Dordrecht: Springer Netherlands.

Dubiel, H. (2001): Kritische Theorie der Gesellschaft: eine einführende Rekonstruktion von den Anfängen im Horkheimer-Kreis bis Habermas. Weinheim: Juventa.

Haas, J. (2019): Negative Öffentlichkeit: Angriffe auf die Gender Studies als Reaktion auf eine erfolgreiche Public Sociology? In: Dörre, K./Haas, J./Ibrahim, W./Petersen, D.J./Richter, K. (Hg.): Im gesellschaftlichen Interesse: Potenziale einer öffentlichen Soziologie. Hamburg: VSA, 101-126.

Healy, K. (2017): Public Sociology in the Age of Social Media. Perspectives on Politics, 15 (3), 771-780.

Heitmeyer, W./Freiheit, M./Sitzer, P. (2020): Rechte Bedrohungsallianzen: Signaturen der Bedrohung II. Berlin: Suhrkamp.

Horkheimer, M. (1931/2009): Die gegenwärtige Lage der Sozialphilosophie und die Aufgaben eines Instituts für Sozialforschung. In: Ders.: Gesammelte Schriften Bd. 3. Frankfurt/M.: Fischer Verlag, 20-35.

Horkheimer, M. (1937/2011): Traditionelle und kritische Theorie: Fünf Aufsätze. 7. Auflage. Frankfurt/M.: Fischer Verlag.

Jeffries, V. (2009): Handbook of public sociology. Lanham, Md: Rowman \& Littlefield Publishers.

Lamla, J. (2014): Öffentlichkeit: Soziologie, Zeitdiagnose und Gesellschaftskritik. In: Lamla, J./Rosa, H./Strecker, D. (Hg.): Handbuch der Soziologie. Konstanz: UVK Verlagsgesellschaft, 491-506.

Lessenich, S. (2014): Soziologie - Krise - Kritik: Zu einer kritischen Soziologie der Kritik. Soziologie, 43 (1), 7-24.

Lessenich, S. (2016): Vorwort. In: Mills, C. W. (1959/2016): Soziologische Phantasie. Wiesbaden: Springer Fachmedien Wiesbaden, 7-21.

Lichtblau, K. (2017): Soziologie und Zeitdiagnose. Oder: Die Moderne im Selbstbezug. In: ders. (Hg.): Zwischen Klassik und Moderne. Die Modernität klassischer deutscher Soziologie. Wiesbaden: Springer VS, 57-79.

Marcuse, H. (1967/2014): Der eindimensionale Mensch: Studien zur Ideologie der fortgeschrittenen Industriegesellschaft. Springe: Zuklampen.
Mau, S. (2017): Das metrische Wir: über die Quantifizierung des Sozialen. Berlin: Suhrkamp.

Mills, C. W. (1960): Images of man: The classic tradition in sociological thinking. New York: G. Braziller.

Mills, C. W. (1959/2016): Soziologische Phantasie. Wiesbaden: Springer Fachmedien Wiesbaden.

Mouffe, C. (2018): Für einen linken Populismus. Berlin: Suhrkamp.

Nachtwey, O. (2016): Die Abstiegsgesellschaft: Über das Aufbegehren in der regressiven Moderne. Berlin: Suhrkamp.

Nassehi, A. (2020): Das große Nein: Eigendynamik und Tragik gesellschaftlichen Protests. Hamburg: Kursbuch.Edition.

Neun, O. (2016): Zur Theorie der soziologischen Gesellschaftsdiagnose. In: Staubmann, H. (Hg.): Soziologie in Österreich - Internationale Verflechtungen. Innsbruck: innsbruck university press.

Neun, O. (2018): Öffentliche Soziologie. Baden-Baden: Nomos.

Neun, O. (2019): Zur Aktualität von C. Wright Mills: Einführung in sein Werk. Wiesbaden: Springer Fachmedien Wiesbaden.

Nymoen, O./Schmitt, W. M. (2021): Influencer: Die Ideologie der Werbekörper. Berlin: Suhrkamp.

Osrecki, F. (2011): Die Diagnosegesellschaft: Zeitdiagnostik zwischen Soziologie und medialer Popularität. Bielefeld: Transcript Verlag.

Osrecki, F. (2018): Die Geschichte der Gegenwartsdiagnostik in der deutschsprachigen Soziologie. In: Moebius, S./ Ploder, A. (Hg.): Handbuch Geschichte der deutschsprachigen Soziologie: Band 1: Geschichte der Soziologie im deutschsprachigen Raum. Wiesbaden: Springer Fachmedien, 453-475.

Reckwitz, A. (2019): Die Gesellschaft der Singularitäten. Berlin: Suhrkamp.

Reif, M. (2016): Professionelle und öffentliche Soziologie: Ein soziologiegeschichtlicher Beitrag zur Professionalisierung der Disziplin in Deutschland. Soziologie, 45 (1), 7-23.

Rosa, H. (2016): Resonanz: Eine Soziologie der Weltbeziehung. Berlin: Suhrkamp.

Schneider, C. J. (2014): Social media and e-public sociology. In: Schneider, C./Hanemaayer, A. (Hg.): The public sociology debate: ethics and engagement. Vancouver: UBC Press, 205-224.

Schwandt, M. (2010): Kritische Theorie: eine Einführung. Stuttgart: Schmetterling Verlag.

Schwarz, L./Wetzel, J. (2019): Feuilleton von unten: Warum die Soziologie mehr Podcasts braucht. Soziologiemagazin, 12 (1), 78-84.

Selke, S. (2020): Einladung zur öffentlichen Soziologie: Eine postdisziplinäre Passion. Wiesbaden: Springer Fachmedien Wiesbaden.

Shrum, W./Castle, L. (2014): „Visionary“ Sociology: Diversions of Public Sociology and Audiovisual Solutions. The American Sociologist, 45 (4), 412-431.
I/IOMENTUM QUARTERLY IIIIIIIIIIIIIIIIIIII

97 
Streeck, W. (2015): Gekaufte Zeit: Die vertagte Krise des demokratischen Kapitalismus. Berlin: Suhrkamp.

Than, N. (2019): Public Sociology: Podcast as an Effective Medium. Online: https://ngathanblog.wordpress. com/2019/02/21/public-sociology-podcast/ [09.01.2021].

Treibel, A./Selke, S. (2012): Soziologie für die Öffentlichkeit. Soziologie, 41 (4), 398-421.

Vobruba, G. (2013): Soziologie und Kritik: Moderne Sozialwissenschaft und Kritik der Gesellschaft. Soziologie, 42 (2), 147-168.

Volkmann, U. (2015): Soziologische Zeitdiagnostik: Eine wissenssoziologische Ortsbestimmung. Soziologie, 44 (2), 139-152.

Wade, L./Sharp, G. (2013): Sociological Images: Blogging as Public Sociology. Social Science Computer Review, 31 (2), 221-28. 